women and 20\% (95\% CI:6-39\%) for girls in the Eastern Mediterranean.

Conclusion There is large global variation in FGM, with the Eastern Mediterranean region recording the highest prevalence. Reassuringly, FGM is lower among girls, highlighting a declining trend in the practice. Some included reports may have underestimated FGM prevalence in girls due to continued risk; future studies should use age cohort analyses to monitor decline. Policy and community-level interventions are needed to meet SDG target 5.3.

\section{OP23 PARENTAL CONSENT FOR TIME-CRITICAL NEONATAL TRIALS IN LOW AND MIDDLE-INCOME COUNTRIES: IS IT TRULY INFORMED?}

${ }^{1}$ Stuti Pant*, ${ }^{2}$ Maya Annie Elias, ${ }^{3}$ Kerry Woolfall, ${ }^{1}$ Sudhin Thayyil. ${ }^{1}$ Centre for Perinatal Neuroscience, Imperial College London, London, UK; ${ }^{2}$ Perinatal Trials Unit Foundation, Bangalore, India; ${ }^{3}$ Institute of Population Health, University of Liverpool, Liverpool, UK

\subsection{6/jech-2021-SSMabstracts.23}

Background Parental consent rates for neonatal interventional trials are significantly higher in Low and middle-income countries (LMIC) than in high-income countries, raising concerns about the credibility of the consent processes (Patterson et al PLOS One 2021). We conducted a mixed-methods study to understand the informed consent process in a neonatal cooling trial [Hypothermia for encephalopathy in low and middleincome countries (HELIX) trial] conducted in India, Sri Lanka and Bangladesh.

Methods Term infants with neonatal encephalopathy, aged less than six hours were randomly allocated to cooling therapy or usual care, following informed parental consent. The consenting process was audio-video $(\mathrm{A}-\mathrm{V})$ recorded in all cases. We analysed the A-V records of the consent process using a 5point Likert scale on three parameters - Empathy, Information, Autonomy. Additionally, we used exploratory observation method to capture relevant aspects of consent process and discussions between parents and professionals. Finally, we conducted in-depth interviews with a subgroup of 20 parents and 15 health care professionals. A thematic analysis was performed on the observations of $\mathrm{A}-\mathrm{V}$ records and on the interview transcripts.

Results In HELIX trial, a total of 475 parents were approached, of which $408(86 \%)$ consented. Of these, 294 $\mathrm{A}-\mathrm{V}$ records were analysed. Median (Interquartile range) score for empathy, information, autonomy was 5 (0), 5 (1) and 5 (1) respectively. However, thematic analysis suggested that the parental decision to participate was based on a unreserved trust in the treating doctors, therapeutic misconception, and access to an expensive treatment free of cost. Most parents did not understand the concept of a clinical trial, nor the nature of the intervention. Lower levels of parental education and misinformation further convoluted the voluntary informed consent process. Parents were visibly incapacitated, and many told the doctor to do whatever is best for the baby. Clinicians lacked equipoise and were biased towards cooling therapy as it was already a standard of care in high-income countries, and this influenced parental decision making. However, the HELIX trial results subsequently showed cooling was harmful and increased mortality in these settings.

Conclusion Despite rigorous research governance and consent process, parental decisions were heavily influenced by situational incapacity and a trust in doctors to make the right decision on their behalf. Further research is required to identify culturally and context appropriate strategies to ensure truly informed trial participation.

\section{OP24 HEALTH IMPACTS, SURVIVAL, QUALITY-ADJUSTED LIFE YEARS, AND COSTS OF CHRONIC KIDNEY DISEASE IN CHILEAN ADULTS}

${ }^{1}$ Magdalena Walbaum*, 'Shaun Scholes, ${ }^{2}$ Ruben Rojas, 'Jennifer Mindell, ${ }^{3}$ Elena Pizzo. ${ }^{1}$ Research Department of Epidemiology and Public Health, University College London, London, UK; ${ }^{2}$ School of Health and Related Research, University of Sheffield, UKi ${ }^{3}$ Department of Applied Health Research, University College London, London, UK

\subsection{6/jech-2021-SSMabstracts.24}

Background Chronic Kidney Disease (CKD), a leading public health problem, poses substantial burdens for both healthcare systems and patients. The aim of this study was to estimate the health and economic burden of CKD for adults aged $40+$ years from the perspective of the Chilean public healthcare system, by adapting the Schlackow and colleagues' CKD-cardiovascular disease (CVD) model.

Methods The microsimulation CKD-CVD model was built based on the Study of Heart and Renal Protection (SHARP). We adapted it using Chilean data, and combining two submodels. The CKD submodel included five mutually exclusive states replicating CKD progression based on the Kidney Disease: Improving Global Outcomes (KDIGO) classification: from CKD stage $3 b$ to end-stage kidney disease (ESKD). The CVD submodel used the individuals' annual risks of cardiovascular outcomes (both fatal and non-fatal) and non-vascular death. Both submodels were combined into a first-order Markov model with annual cycles to allow the inclusion of all the possible states between the CKD stages and the CVD outcomes. We used nationally-representative Chilean survey and registry data to adapt the model with a time horizon of 20 years, from 2020 to 2040. Costs, life-years (Lys), and qualityadjusted life-years (QALYs) were discounted using a 3\% discount rate after the first year.

Results On average, an individual aged 64 years, starting with CKD stage $3 \mathrm{~b}$ at baseline, had a projected cumulative probability of progressing to ESKD of 0.16 (95\% credibility interval CI: $0.13-0.20)$; and projected survival of 11.9 years $(95 \% \mathrm{CI}$ : 11.2-12.5) and 8.9 QALYs (95\% CI: 8.3-9.5). An individual starting in ESKD and having renal replacement therapy had projected survival of 7.7 years (95\% CI: 7.0-8.4) and 5.7 QALYs (95\% CI: 5.1-6.2). For individuals in CKD Stage 3b, the predicted probabilities of having a major vascular event (MVE) or vascular death (VD) were 0.14 (0.11-0.18), 0.26 $(0.21-0.33)$ and $0.43(0.35-0.54)$ in the next 5, 10 and 20 years, respectively. For individuals in ESKD, the predicted probabilities of having a MVE or VD over the same time periods were $0.31(0.26-0.37), 0.46(0.39-0.53)$ and 0.59 (0.51-0.68); mean lifetime direct healthcare costs were $£ 70,606$ (95\% CI: 57,385-85,190).

Conclusion The important interdependence between CKD and CVD outcomes and the lower life expectancy and quality-adjusted survival when individuals progress to more advanced stages of the disease highlight the need for effective public health policies to address the management of the disease and its risk factors at primary and secondary healthcare level. 